\title{
SCHÜLERLEISTUNGEN IM INTERNATIONALEN VERGLEICH
}

Wie gut gelingt es den Bildungssystemen, jungen Menschen ein solides Fundament an Kenntnissen und Fähigkeiten zu vermitteln, mit dem sie für das Leben und für die Fortsetzung des Lernprozesses über die Schule hinaus gerüstet sind? Die Internationale OECD-Schulleistungsstudie (PISA) evaluiert die Kenntnisse und Fertigkeiten, die Schülerinnen und Schüler im Alter von 15 Jahren, d.h. gegen Ende ihrer Pflichtschulzeit besitzen. Der Schwerpunkt der PISA-Erhebung 2009 lag auf der Lesekompetenz, doch wurde erstmals auch die Fähigkeit der Schülerinnen und Schüler untersucht, digitale Texte zu lesen, zu verstehen und zu nutzen.

\section{Definition}

Die PISA-Erhebung erstreckt sich auf die Bereiche Lesekompetenz, Mathematik und Naturwissenschaften. In der PISA-Erhebungsrunde 2009 entfiel eine Stunde Testzeit auf den Bereich Lesekompetenz, eine halbe Stunde auf Mathematik und eine halbe Stunde auf Naturwissenschaften. Die teilnehmenden Schüler verbrachten jeweils zwei Stunden mit der Bearbeitung der Testaufgaben. In 19 Ländern wurden den Schülerinnen und Schülern zusätzliche, am Computer zu lösende Fragen gestellt, um ihre Fähigkeit zum Lesen digitaler Texte zu testen.

Lesekompetenz wird definiert als die Fähigkeit, geschriebene Texte zu verstehen, zu nutzen und über sie zu reflektieren, um eigene Ziele zu erreichen, das eigene Wissen und Potenzial weiterzuentwickeln und am gesellschaftlichen Leben teilzunehmen. Mathematische Grundbildung wird definiert als die Fähigkeit, die Rolle der Mathematik in der Welt zu erkennen und zu verstehen, wohlfundierte Urteile abzugeben und Mathematik in einer Weise

\section{Überblick}

Aus den Abbildungen ist die Differenz zwischen dem OECD-Durchschnittsergebnis im Bereich Lesekompetenz (493 Punkte) und den Mittelwerten der einzelnen Länder ersichtlich. Wie bereits in der PISA-Erhebung 2006 übertrifft Korea im Bereich Lesekompetenz alle anderen teilnehmenden OECD-Länder. In den Vereinigten Staaten, Schweden, Deutschland, Irland, Frankreich, Dänemark, im Vereinigten Königreich, in Ungarn und Portugal weichen die Ergebnisse im Bereich Lesekompetenz nicht signifikant vom OECD-Durchschnitt ab. Die Abbildung zeigt auch die Mathematikergebnisse im Vergleich zum OECDDurchschnittswert (496 Punkte). Die meisten Länder, die in einem Bereich gute Ergebnisse erzielen, tun dies zwar auch in den anderen Bereichen, in einigen Ländern sind jedoch erhebliche Unterschiede festzustellen: So erzielt z.B. die Schweiz in Mathematik bessere Ergebnisse als im Bereich Lesekompetenz.

Die Tabelle zeigt die geschlechtsspezifischen Ergebnisse. Wie bereits in PISA 2006 erzielen die Mädchen im Bereich Lesekompetenz in allen Ländern signifikant bessere Ergebnisse als die Jungen; im Durchschnitt ist eine Differenz von 39 Punkten zu beobachten. Umgekehrt schneiden die Jungen in Mathematik besser ab als die Mädchen, wobei die durchschnittliche Differenz 12 Punkte beträgt. In Naturwissenschaften sind im Durchschnitt keine geschlechtsspezifischen Leistungsunterschiede festzustellen, in einigen Ländern weichen die Ergebnisse von Mädchen und Jungen jedoch deutlich voneinander ab. Zum Beispiel schneiden in den Vereinigten Staaten Jungen in Naturwissenschaften signifikant besser ab als Mädchen, während es in Finnland umgekehrt ist. zu nutzen, die den Anforderungen engagierter und reflektierender Bürger gerecht wird. Naturwissenschaftliche Grundbildung wird definiert als die Fähigkeit, naturwissenschaftliches Wissen anzuwenden, um Fragestellungen zu identifizieren, neue Erkenntnisse zu erwerben, naturwissenschaftliche Phänomene $\mathrm{zu}$ erklären und auf Beweisen basierende Schlüsse über naturwissenschaftliche Sachverhalte zu ziehen.

Der Mittelwert der ursprünglichen PISA-Gesamtskalen Lesekompetenz (Schwerpunktbereich der PISA-Erhebung 2000), Mathematik (Schwerpunktbereich der PISA-Erhebung 2003) und Naturwissenschaften (Schwerpunktbereich der PISA-Erhebung 2006) wurde für die teilnehmenden OECD-Länder auf 500 gesetzt, da etwa zwei Drittel der Schülerinnen und Schüler in den OECDLändern zwischen 400 und 600 Punkte erzielten. In der PISA-Studie 2009, an der ein etwas breiteres Spektrum von OECD-Ländern teilnahm, veränderte sich der Mittelwert für jeden Bereich: 493 Punkte für den Bereich Lesekompetenz, 496 für den Bereich Mathematik und 501 für den Bereich Naturwissenschaften.

\section{Vergleichbarkeit}

Bei der Bestimmung von Umfang und Art der Leistungsmessung werden führende Fachleute in den PISA-Teilnehmerstaaten zu Rate gezogen, wobei die endgültigen Entscheidungen von den zuständigen staatlichen Stellen der OECD-Länder getroffen werden. Es werden erhebliche Ressourcen und Anstrengungen darauf verwendet, bei dem Erhebungsmaterial kulturelle und sprachliche Breite sowie Ausgewogenheit zu gewährleisten. Für Übersetzungsverfahren, Stichprobenauswahl und Datenerhebung werden strenge Mechanismen der Qualitätssicherung eingesetzt.

Im Rahmen von PISA 2009 wurden über 520000 15-jährige Schülerinnen und Schüler in 75 Teilnehmerländern bzw. -volkswirtschaften evaluiert. Da die Ergebnisse auf Wahrscheinlichkeitsstichproben beruhen, werden in den Tabellen normalerweise Standardfehler (S.E.) angegeben.

\section{Quelle}

- OECD (2010), PISA 2009 Ergebnisse: Was Schülerinnen und Schüler wissen und können: Schülerleistungen in Lesekompetenz, Mathematik und Naturwissenschaften (Band I), W. Bertelsmann Verlag, Bielefeld.

- OECD (2007), PISA 2006: Naturwissenschaftliche Kompetenzen für die Welt von morgen, W. Bertelsmann Verlag, Bielefeld.

\section{Weitere Informationen}

Analysen

- OECD (2010), PISA 2009 Ergebnisse (Veröffentlichungsreihe), OECD Publishing.

- OECD (2012), Bildung auf einen Blick, W. Bertelsmann Verlag, Bielefeld.

- OECD (2012), Highlights from Education at a Glance, OECD Publishing.

\section{Statistiken}

- OECD (2010), PISA 2009 at a Glance, OECD Publishing.

\section{Zur Methodik}

- OECD (2009), PISA 2009 Assessment Framework: Key Competencies in Reading, Mathematics and Science, PISA, OECD Publishing.

\section{Online-Datenbanken}

- OECD PISA Database.

Websites

- Internationale Schulleistungsstudie der OECD (PISA), www.pisa.oecd.org. 
Durchschnittsergebnisse in PISA 2009 nach Geschlecht

2009

\begin{tabular}{|c|c|c|c|c|c|c|c|c|c|c|c|c|}
\hline & \multicolumn{4}{|c|}{ Lesekompetenz } & \multicolumn{4}{|c|}{ Mathematik } & \multicolumn{4}{|c|}{ Naturwissenschaften } \\
\hline & \multicolumn{2}{|c|}{ Mädchen } & \multicolumn{2}{|c|}{ Jungen } & \multicolumn{2}{|c|}{ Mädchen } & \multicolumn{2}{|c|}{ Jungen } & \multicolumn{2}{|c|}{ Mädchen } & \multicolumn{2}{|c|}{ Jungen } \\
\hline & Mittelwert & S.E. & Mittelwert & S.E. & Mittelwert & S.E. & Mittelwert & S.E. & Mittelwert & S.E. & Mittelwert & S.E. \\
\hline Australien & 533 & 2.6 & 496 & 2.9 & 509 & 2.8 & 519 & 3.0 & 528 & 2.8 & 527 & 3.1 \\
\hline Belgien & 520 & 2.9 & 493 & 3.4 & 504 & 3.0 & 526 & 3.3 & 503 & 3.2 & 510 & 3.6 \\
\hline Chile & 461 & 3.6 & 439 & 3.9 & 410 & 3.6 & 431 & 3.7 & 443 & 3.5 & 452 & 3.5 \\
\hline Dänemark & 509 & 2.5 & 480 & 2.5 & 495 & 2.9 & 511 & 3.0 & 494 & 2.9 & 505 & 3.0 \\
\hline Deutschland & 518 & 2.9 & 478 & 3.6 & 505 & 3.3 & 520 & 3.6 & 518 & 3.3 & 523 & 3.7 \\
\hline Estland & 524 & 2.8 & 480 & 2.9 & 508 & 2.9 & 516 & 2.9 & 528 & 3.1 & 527 & 3.1 \\
\hline Finnland & 563 & 2.4 & 508 & 2.6 & 539 & 2.5 & 542 & 2.5 & 562 & 2.6 & 546 & 2.7 \\
\hline Frankreich & 515 & 3.4 & 475 & 4.3 & 489 & 3.4 & 505 & 3.8 & 497 & 3.5 & 500 & 4.6 \\
\hline Griechenland & 506 & 3.5 & 459 & 5.5 & 459 & 3.3 & 473 & 5.4 & 475 & 3.7 & 465 & 5.1 \\
\hline Irland & 515 & 3.1 & 476 & 4.2 & 483 & 3.0 & 491 & 3.4 & 509 & 3.8 & 507 & 4.3 \\
\hline Island & 522 & 1.9 & 478 & 2.1 & 505 & 1.9 & 508 & 2.0 & 495 & 2.0 & 496 & 2.1 \\
\hline Israel & 495 & 3.4 & 452 & 5.2 & 443 & 3.3 & 451 & 4.7 & 456 & 3.2 & 453 & 4.4 \\
\hline Italien & 510 & 1.9 & 464 & 2.3 & 475 & 2.2 & 490 & 2.3 & 490 & 2.0 & 488 & 2.5 \\
\hline Japan & 540 & 3.7 & 501 & 5.6 & 524 & 3.9 & 534 & 5.3 & 545 & 3.9 & 534 & 5.5 \\
\hline Kanada & 542 & 1.7 & 507 & 1.8 & 521 & 1.7 & 533 & 2.0 & 526 & 1.9 & 531 & 1.9 \\
\hline Korea & 558 & 3.8 & 523 & 4.9 & 544 & 4.5 & 548 & 6.2 & 539 & 4.2 & 537 & 5.0 \\
\hline Luxemburg & 492 & 1.5 & 453 & 1.9 & 479 & 1.3 & 499 & 2.0 & 480 & 1.6 & 487 & 2.0 \\
\hline Mexiko & 438 & 2.1 & 413 & 2.1 & 412 & 1.9 & 425 & 2.1 & 413 & 1.9 & 419 & 2.0 \\
\hline Neuseeland & 544 & 2.6 & 499 & 3.6 & 515 & 2.9 & 523 & 3.2 & 535 & 2.9 & 529 & 4.0 \\
\hline Niederlande & 521 & 5.3 & 496 & 5.1 & 517 & 5.1 & 534 & 4.8 & 520 & 5.9 & 524 & 5.3 \\
\hline Norwegen & 527 & 2.9 & 480 & 3.0 & 495 & 2.8 & 500 & 2.7 & 502 & 2.8 & 498 & 3.0 \\
\hline Österreich & 490 & 4.0 & 449 & 3.8 & 486 & 4.0 & 506 & 3.4 & 490 & 4.4 & 498 & 4.2 \\
\hline Polen & 525 & 2.9 & 476 & 2.8 & 493 & 3.2 & 497 & 3.0 & 511 & 2.8 & 505 & 2.7 \\
\hline Portugal & 508 & 2.9 & 470 & 3.5 & 481 & 3.1 & 493 & 3.3 & 495 & 3.0 & 491 & 3.4 \\
\hline Schweden & 521 & 3.1 & 475 & 3.2 & 495 & 3.3 & 493 & 3.1 & 497 & 3.2 & 493 & 3.0 \\
\hline Schweiz & 520 & 2.7 & 481 & 2.9 & 524 & 3.4 & 544 & 3.7 & 512 & 3.0 & 520 & 3.2 \\
\hline Slowak. Rep. & 503 & 2.8 & 452 & 3.5 & 495 & 3.4 & 498 & 3.7 & 491 & 3.2 & 490 & 4.0 \\
\hline Slowenien & 511 & 1.4 & 456 & 1.6 & 501 & 1.7 & 502 & 1.8 & 519 & 1.6 & 505 & 1.7 \\
\hline Spanien & 496 & 2.2 & 467 & 2.2 & 474 & 2.5 & 493 & 2.3 & 485 & 2.3 & 492 & 2.5 \\
\hline Tschech. Rep. & 504 & 3.0 & 456 & 3.7 & 490 & 3.0 & 495 & 3.9 & 503 & 3.2 & 498 & $4 . C$ \\
\hline Türkei & 486 & 4.1 & 443 & 3.7 & 440 & 5.6 & 451 & 4.6 & 460 & 4.5 & 448 & 3.8 \\
\hline Ungarn & 513 & 3.6 & 475 & 3.9 & 484 & 3.9 & 496 & 4.2 & 503 & 3.5 & 503 & 3.8 \\
\hline Ver. Königreich & 507 & 2.9 & 481 & 3.5 & 482 & 3.3 & 503 & 3.2 & 509 & 3.2 & 519 & 3.6 \\
\hline Ver. Staaten & 513 & 3.8 & 488 & 4.2 & 477 & 3.8 & 497 & 4.0 & 495 & 3.7 & 509 & 4.2 \\
\hline EU27 & .. & .. & .. & & .. & .. & .. & .. & .. & .. & .. & . \\
\hline OECD & 513 & 0.5 & 474 & 0.6 & 490 & 0.6 & 501 & 0.6 & 501 & 0.6 & 501 & 0.6 \\
\hline Brasilien & 425 & 2.8 & 397 & 2.9 & 379 & 2.6 & 394 & 2.4 & 404 & 2.6 & 407 & 2.6 \\
\hline China & .. & .. & .. & .. & .. & .. & .. & .. & .. & .. & .. & . \\
\hline Indien & .. & .. & .. & .. & .. & .. & .. & .. & .. & .. & .. & . \\
\hline Indonesien & 420 & 3.9 & 383 & 3.8 & 372 & 4.0 & 371 & 4.1 & 387 & 4.0 & 378 & $4.2 . r u$ \\
\hline Russ. Föderation & 482 & 3.4 & 437 & 3.6 & 467 & 3.5 & 469 & 3.7 & 480 & 3.5 & 477 & 3.7 \\
\hline Südafrika & .. & .. & .. & .. & .. & .. & .. & .. & .. & .. & .. & . \\
\hline
\end{tabular}

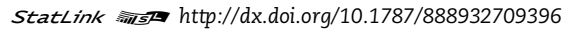

\section{Ergebnisse in den Bereichen Lesekompetenz und Mathematik in PISA 2009}

Mittelwert

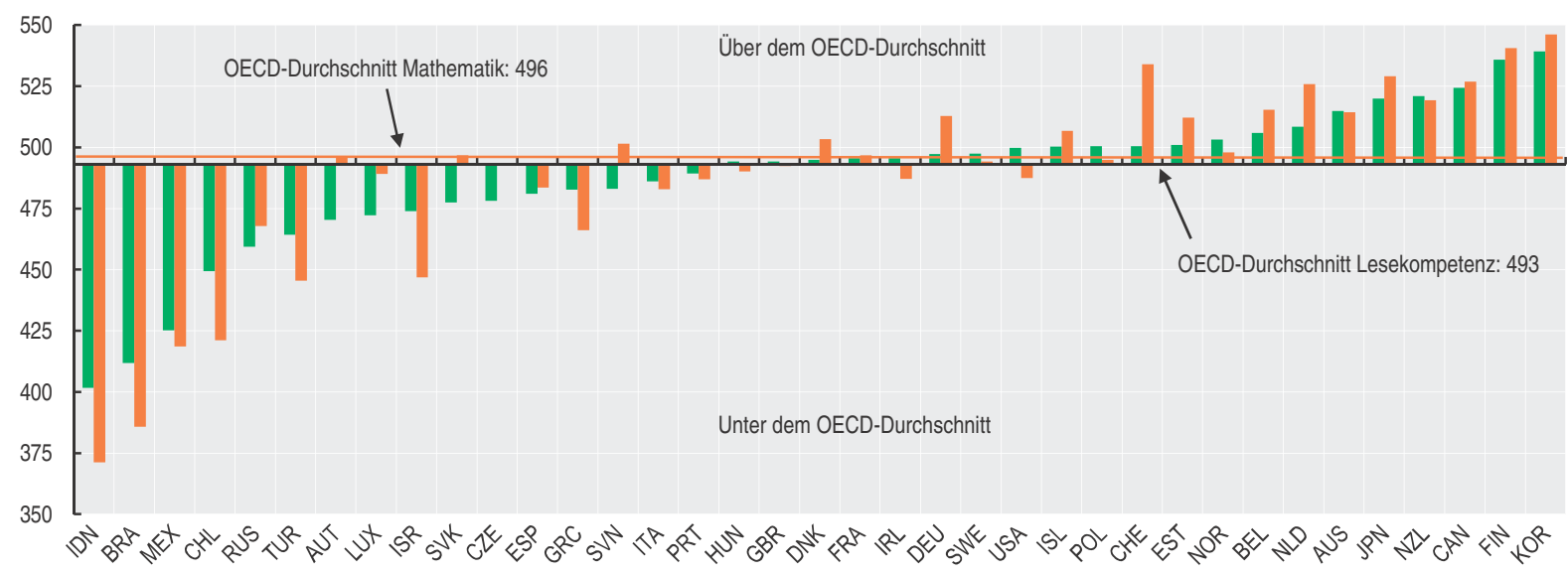




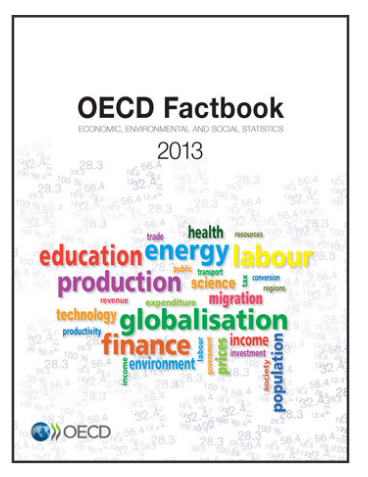

\section{From: \\ OECD Factbook 2013 \\ Economic, Environmental and Social Statistics}

Access the complete publication at:

https://doi.org/10.1787/factbook-2013-en

\section{Please cite this chapter as:}

OECD (2013), "Schülerleistungen im internationalen Vergleich", in OECD Factbook 2013: Economic, Environmental and Social Statistics, OECD Publishing, Paris.

DOI: https://doi.org/10.1787/factbook-2013-72-de

Das vorliegende Dokument wird unter der Verantwortung des Generalsekretärs der OECD veröffentlicht. Die darin zum Ausdruck gebrachten Meinungen und Argumente spiegeln nicht zwangsläufig die offizielle Einstellung der OECDMitgliedstaaten wider.

This document and any map included herein are without prejudice to the status of or sovereignty over any territory, to the delimitation of international frontiers and boundaries and to the name of any territory, city or area.

You can copy, download or print OECD content for your own use, and you can include excerpts from OECD publications, databases and multimedia products in your own documents, presentations, blogs, websites and teaching materials, provided that suitable acknowledgment of OECD as source and copyright owner is given. All requests for public or commercial use and translation rights should be submitted to rights@oecd.org. Requests for permission to photocopy portions of this material for public or commercial use shall be addressed directly to the Copyright Clearance Center (CCC) at info@copyright.com or the Centre français d'exploitation du droit de copie (CFC) at contact@cfcopies.com. 